\title{
Antibodies to streptococcal inhibitor of complement function and $M$ peptides in a post-streptococcal glomerulonephritis endemic region of Australia.
}

\author{
K. S. SRIPRAKASH*+†, JON HARTAS* and ANDREW WHITE* $\S$ \\ * Menzies School of Health Research, Darwin; †Co-operative Research Centre for Aboriginal and Tropical \\ Health, Darwin, $\$$ Queensland Institute of Medical Research, Brisbane and §Remote Health Service, Alice \\ Springs
}

\begin{abstract}
Post-streptococcal glomerulonephritis (PSGN) is an immune-mediated disease in which an immune complex containing a streptococcal antigen are deposited in affected glomeruli. Strains of only some $M$ types are known to be associated with PSGN. A secretory protein called SIC inhibits complement function. Whereas all M1 and M57 strains express closely related SIC (CRS), all M12 and M55 strains express distantly related SIC (DRS) proteins. Strains belonging to these four $M$ types are historically associated with PSGN. This study used ELISA to analyse 112 sera from individuals with a recorded history of PSGN and 86 sera from individuals who had no such recorded history, all from a PSGN endemic region in tropical Australia. Antibody reactions to CRS, DRS and peptides corresponding to the N-termini of M1, M5, M12, M49, M55 and M57 antigens were assessed. A large proportion of the population showed reactions to each of these antigens and there was no correlation between CRS seropositivity and antibodies to CRS-positive $M$ types. Likewise there was no correlation between DRS seropositivity and antibodies to DRS-positive $M$ types. Interestingly, in this community endemic for PSGN a significantly higher proportion of DRS seropositive subjects had a recorded history of PSGN than did DRS seronegative subjects. DRS may have a predictive value for PSGN diagnosis or a role in PSGN pathogenesis.
\end{abstract}

\section{Introduction}

Streptococcus pyogenes (Lancefield group A streptococcus, GAS) is an aetiological agent for pharyngitis and pyoderma. An important sequela of GAS infection is post-streptococcal glomerulonephritis (PSGN) [1], which has been shown to be associated with long-term renal dysfunction [2]. $M$ protein is a major GAS surface antigen, which elicits a protective immune response, that is persistent for some $M$ proteins [3]. Only certain $M$ serotypes have been shown to be associated with PSGN $[1,4,5]$.

Serotype 1 strains of $S$. pyogenes express a secretory protein, that inhibits complement-mediated cell lysis [6]. The gene for this streptococcal inhibitor of

Received 29 Oct. 2001; revised reversion received 9 Jan. 2002; accepted 14 Jan. 2002.

Corresponding author: Dr K.S. Sriprakash (e-mail: sriS (a)imr.edu.au). complement function (SIC) has been found only among $\mathrm{M}$ types 1 and 57 of $55 \mathrm{M}$ types tested. SIC from strain AP1, an M1 strain, has been shown to bind to clusterin and to be incorporated into the complement membrane attack complex (MAC). Subsequently, two classes of SIC were described - those closely related to the SIC from AP1 (CRS) and those distantly related to the SIC from AP1 (DRS) [7]. Both CRS and DRS have a similar leader sequence and show $85 \%$ similarity in a stretch of 55 residues in the C-proximal half of the 25$35-\mathrm{kDa}$ protein; otherwise, CRS and DRS share little sequence similarity.

All strains containing emm 1 (gene for M1 protein) and emm57 possess CRS genes (crs 1 and crs57, respectively), whereas emm 12 and emm55 strains have DRS genes ( $d r s 12$ and $d r s 55$, respectively). Moreover, in emm 1 , emm 12 and emm55 strains, the crs $1, d r s 12$ and $d r s 55$ are located immediately downstream from their respective emm locus and form a part of the $m g a$ regulon [8]. In emm57 strains, crs57 is outside the $m g a$ 
regulon. The CRS- and DRS-expressing $\mathrm{M}$ types are historically associated with PSGN [1].

PSGN is an immune-mediated disease $[9,10]$. Immune complexes containing a streptococcal antigen are deposited in the glomeruli of PSGN patients. Only $S$. pyogenes $\mathrm{M}$ types historically associated with PSGN express such an antigen. The following were reported to have a role in the pathogenesis of PSGN [10-15]: (i) one or more streptococcal extracellular products including $\mathrm{M}$ protein, (ii) a nephritis strain-associated protein, (iii) a pre-absorbing antigen, (iv) a nephritisassociated plasmin receptor (NAPlr) and (v) a zymogen encoded by SpeB gene. Parra et al [14] compared the antibody response to streptococcal cysteine protease zymogen, streptolysin O and DNAase B in populations from three South American countries. Although they found that anti-zymogen titres were consistently higher in the PSGN patients than in healthy controls, there was no significant difference between PSGN patients and a control group with uncomplicated impetigo. Similarly, antibodies to NAPlr were found in a significantly greater proportion of PSGN patients than in control groups [13]. Age-related acquisition of NAPlr antibodies was noted, possibly resulting from repeated streptococcal infections. These antibodies appear to be long lasting.

Rodríquez-Iturbe [16] described three criteria for potential nephritogenic antigens. They are that the antigen (i) is secreted only by nephritis-associated GAS strains, (ii) is present in glomeruli of PSGN patients, and (iii) induces antibodies in convalescent sera from PSGN patients. SIC fulfils these criteria as it is a secretory product restricted to $\mathrm{M}$ types that are historically associated with PSGN, it is highly immunogenic [17], and through its interaction with MAC and clusterin [6] SIC has a potential to be located in affected glomeruli. To test the hypothesis that SIC or its variants play a role in the pathogenesis of PSGN, this study investigated relationships between antibodies against CRS/DRS, antibodies against the CRS/DRSpositive $\mathrm{M}$ types, and history of clinical PSGN in a population from an island community in the Northern Territory of Australia where streptococcal infection and PSGN are endemic [18].

\section{Materials and methods}

\section{Strain and peptides}

S. pyogenes M type 1 strain 2031 was obtained from the Group A Streptococcus Reference Laboratory in Prague, Czech Republic. NS488 is a Northern Territory isolate of emm 12 sequence type. Peptides corresponding to M1, M5, M12, M49, M55 and M57 were obtained from Mimotopes, Australia (Table 1). The 15 mer peptides were extended by GSG residues and were biotinylated at the $\mathrm{C}$-terminus.
Table 1. Sequences of biotinylated peptides representing the N-terminal region of mature $M$ proteins

\begin{tabular}{ll}
\hline M peptide & Sequence \\
\hline M1 & NGDGNPREVIEDLAAGSG \\
M5 & AVTRGTINDPQRAKEGSG \\
M12 & DHSDLVAEKQRLEDLGSG \\
M49 & AEKKVEAKVEVAENNGSG \\
M55 & NQTEPSQTNNRLYQEGSG \\
M57 & NDDITSMTPILSGVGGSG \\
\hline
\end{tabular}

\section{Cloning and expression of CRS and DRS}

The crs 1 gene from strain 2031 was amplified with SIC-specific forward and reverse primers (CRSf: GGGGGATCCATGAATATTAGAAATAAGATTGAAA ATAG and CRSr: AAACTAGGATCCTCGCATCCCTT TACGTTGCTG, respectively) in a PCR. The amplified product was cloned into pQE30 (Qiagen) upstream of the His6 tag. Likewise, drs 12 was amplified from NS488 with CRSf and DRSr (GGGCTGCAGGCAA GAATCTTTCTATTTAATACC) as primers. The recombinant CRS and DRS (rCRS and rDRS) were purified from cell lysates of Escherichia coli harbouring appropriate clones with Ni-NTA affinity columns (Qiagen) according to the manufacturer's instructions, with a buffer containing $8 \mathrm{M}$ urea. The eluates from the columns were extensively dialysed against phosphatebuffered saline (PBS). The purified proteins migrated between 30 and $45 \mathrm{kDa}$ as characterised by SDS-PAGE followed by immunoblotting with antibodies raised against rCRS and rDRS in rabbits.

\section{Study population}

Sera were collected from an Australian Aboriginal island community in Northern Australia. Population-based health screening was conducted on the island between 1992 and 1999 and a large proportion of the community participated. Part of the study included review of medical records for history of PSGN and collection of serum. In all, 112 participants had a history of PSGN and their sera were tested along with those from 86 control subjects who had no known history of PSGN. The Joint Institutional Ethics Committee of the Royal Darwin Hospital and the Menzies School of Health Research approved the study, as did the local community health board. Consent was obtained from each individual or guardian at the time of the screening.

\section{ELISA}

Detection of CRS and DRS antibodies in the sera. Flatbottomed immunoplates (Nunc) were coated by adding $100 \mu \mathrm{l}$ of rCRS or rDRS $100 \mu \mathrm{g} / \mathrm{ml}$ in carbonate buffer ( $\mathrm{pH}$ 9.6) per well and incubating overnight at $4^{\circ} \mathrm{C}$. After blocking with denatured casein hydrolysate $5 \%$ in PBS, the bound proteins were allowed to react with a 1 in 300 dilution of human serum in the same buffer containing Tween $200.05 \%$ for $1 \mathrm{~h}$ at room temperature (RT). After washing with Tween $200.05 \%$ 
in PBS and treating with a secondary anti-human IgG antibody conjugated to alkaline phosphatase (Sigma), the reactions were detected with 4-nitrophenyl-disodium orthophosphate. The optical density (OD) was measured at $405 \mathrm{~nm}$ after incubation for $1 \mathrm{~h}$ at room temperature. Sera from four non-Aboriginal laboratory workers who had no recorded history of PSGN showed no reaction to the rCRS or rDRS proteins by these criteria.

Detection of antibodies to peptides corresponding to M1, M5, M12, M49, M55 and M57. Flat-bottomed immunoplates (Nunc) were coated with streptavidin $\left(50 \mu \mathrm{g} / \mathrm{ml}\right.$ in PBS) $100 \mu \mathrm{l} /$ well and incubated at $37^{\circ} \mathrm{C}$ overnight. After blocking as above, biotinylated peptides were added to the wells $(50 \mu \mathrm{l}, 0.66 \mu \mathrm{g} / \mathrm{ml})$. The plates were incubated at room temperature for $1 \mathrm{~h}$. After washing with Tween $200.05 \%$ in PBS, sera (1 in 300 dilution) were added and incubated for $1 \mathrm{~h}$ at RT. The reactions were measured as described above. Sera from four non-Aboriginal laboratory workers were also tested; past infection with one or more GAS strains was evident as two sera exhibited weak reactions with the M1 or M12 peptides. Controls without biotinylated peptides were also included.

\section{Analysis}

Data from a population study such as this are usually analysed by determining seropositivity based on a person having a result $>2$ SD from the mean of results from a large unexposed population. In this study, because OD was not normally distributed (see Results), and normal values have not been established for an unexposed population because of the high endemicity of PSGN, this method could not be used. Therefore, a cut-off value for seropositivity as three times the mean of the lowest quartile of the samples for each antigen was used, as described by Hoe et al. [17]. Fisher's exact test was used to calculate significance levels for relationships between positive serology to various peptides, and with history of PSGN. Statistical calculations were made with Stata 6 software [19].

\section{Results and discussion}

In a cross-sectional study of an island community of approximately 1400 residents, sera from 198 subjects were analysed, representing c. $15 \%$ of the population. Both sexes were equally represented (male/female $=106 / 92)$ and the majority $(78 \%)$ of sera were from individuals older than 15 years (age range $10-58$ years).

In conjunction with testing for seroreaction to CRS and DRS, four M peptides representing the four CRS- and DRS-positive $M$ types, and two $M$ peptides representing two CRS- and DRS-negative $M$ types (M5 and M49) were also tested. Table 2 presents a summary of the distribution of data for the eight antigens tested. The distribution is skewed towards low values. Fig. 1 shows the proportions of sera reactive to CRS, DRS and the $\mathrm{M}$ peptides tested.

In all, 113 sera (57\%) reacted with CRS and 109 (55\%) reacted with DRS; $73 \%$ of the sera reacted with either CRS or DRS and 77 sera (39\%) reacted with both the antigens. The results presented here show that like CRS, DRS proteins are immunogenic in natural infection and $>70 \%$ of the sera from this population have antibodies to either of these antigens. A total of 179 sera $(90 \%)$ reacted with at least one of the six M peptides, $20(10 \%)$ reacted with only one, $80 \%$ reacted with two or more and almost $37 \%$ reacted with all six peptides. These results suggest that most of the study population has been exposed to multiple serotypes. These results are not unexpected given the very high frequency of skin sepsis in the population $[18,20]$ and the high antigenic diversity of circulating GAS strains in this geographical location [21,22].

Rates of seropositivity to the peptides that represent CRS- or DRS-positive M types, or CRS- and DRSnegative $M$ types were similar (Fig. 1). Serology in this study does not reflect the rate of isolation of strains belonging to these $M$ types over the past 10 years; for example, strains with emm 12 or emm 49 sequence were rarely isolated, whereas emm55 types were common. However, the prevalence of specific strains at the time when most participants in this study were children is not known.

There was a positive correlation between the presence of antibodies to CRS and antibodies to DRS $(\mathrm{p}<$ $0.001) ; 68 \%$ of sera showing antibodies to CRS also

Table 2. Summary of distributions of the raw scores of OD in ELISAs

\begin{tabular}{lccccc}
\hline & \multicolumn{5}{c}{ OD $_{405}$} \\
\cline { 2 - 6 } Antigen & Mean & Range & 25th percentile & Median & Cut off* $^{*}$ \\
\hline M1 & 0.11 & $0-0.98$ & 0.04 & 0.09 & 0.05 \\
M57 & 0.12 & $0-1.00$ & 0.05 & 0.09 & 0.08 \\
M12 & 0.11 & $0-1.05$ & 0.03 & 0.08 & 0.04 \\
M55 & 0.16 & $0-0.88$ & 0.05 & 0.08 & 0.07 \\
M5 & 0.11 & $0-0.85$ & 0.04 & 0.09 & 0.05 \\
M49 & 0.11 & $0-0.86$ & 0.04 & 0.08 & 0.05 \\
CRS & 0.18 & $0-2.06$ & 0.06 & 0.11 & 0.06 \\
DRS & 0.12 & $0-1.37$ & 0.03 & 0.06 & 0.10 \\
\hline
\end{tabular}

* The cut-off point used seropositivity was three times the mean of the lowest quartile. 


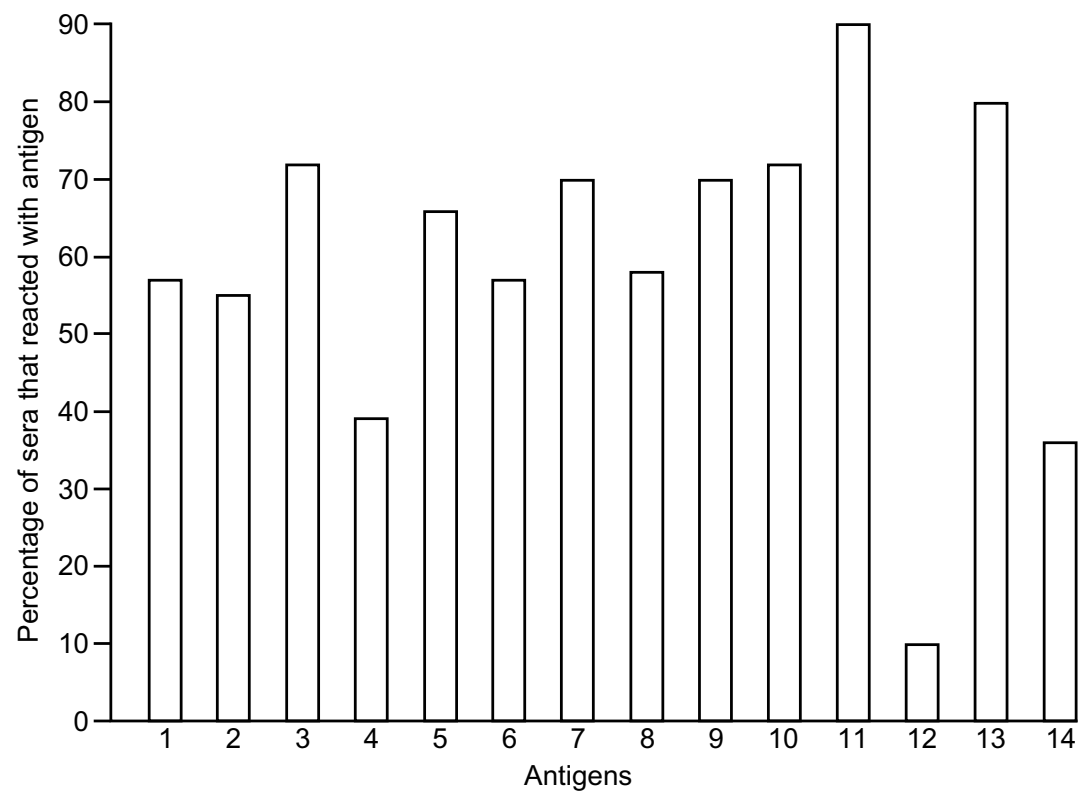

Fig. 1. Proportion of sera that reacted with CRS, DRS and the six M peptides; 198 sera were analysed by ELISA for antibodies to the streptococcal antigens. 1, CRS; 2, DRS; 3, CRS or DRS; 4, CRS + DRS; 5-10, M1, M57, M12, M55, M5 and M49 peptides, respectively; 11, at least one $M$ peptide; 12, only one $M$ peptide; 13, two or more M peptides; 14, all six M peptides. Three times the mean $\mathrm{OD}_{405}$ of the lowest quartile was used for the cut-off value in these ELISAs (see text). The range of these cut-off values for the eight individual antigens tested in this study was $0.04-0.1$. The sequences of peptides are shown in Table 1 . Purified recombinant proteins were used for CRS and DRS.

reacted with DRS, whereas only $38 \%$ of CRS antibodynegative sera reacted with DRS. It is unlikely that sequence variation in CRS [23] would influence the above outcome, as several linear B-cell epitopes spanning the entire length of CRS are immunogenic in natural infection [17] and it is unlikely that the variants exhibit changes in all the epitopes. Taken together, these results suggest that a proportion of the population had been infected previously with both CRS- and DRS-positive $M$ types. This should then reflect in a high prevalence of antibodies to M12 and M55 peptides among the sera containing antibodies to M1 and M57 peptides. As expected, 142 (93\%) of the sera that reacted with the M1 or M57 peptide also reacted with the M12 or M55 peptide, whereas only $26 \%$ of the sera that showed no reactivity with either the M1 or M57 peptide reacted with the M12 or M55 peptide $(p<0.001)$. One explanation of this is that some individuals in this population are more likely than others to have been infected with and mounted an immune response to multiple strains of GAS.

As CRS appears to be restricted to only M1 and M57 serotypes, and DRS appears to be restricted to M12 and M55 types, the observed congruence between the above positive associations (M1/M57 and M12/M55 seroreactions, and CRS and DRS seroreactions) was not surprising. It was also anticipated that CRS seropositivity would be directly associated with seropositivity to M1 and M57 peptide and that DRS seropositivity would be associated with seropositivity to M12 and M55 peptide. However, such associations were not found. Of the 113 CRS-positive sera, 92
(81\%) reacted with M1 or M57 peptides compared with $60(71 \%)$ of CRS-negative sera $(p=0.09)$. Of the 109 DRS-positive sera, 76\% reacted with M12 or M55 peptide; a similar proportion of the DRS-negative sera $(79 \%)$ reacted with these $\mathrm{M}$ peptides $(\mathrm{p}=0.608)$. Two factors may have obscured the relationships between seropositivity to CRS/DRS and seropositivity to the M peptide. First, like M1 antibodies [17], M57, M12 and M55 antibodies may preferentially decline over time relative to CRS/DRS antibodies. Second, because M proteins are surface-bound, early treatment with antibiotics may remove the antigen along with the pathogen and suppress the type-specific antibody response [24], whereas CRS and DRS are secretory products, which may continue to circulate even after the disappearance of infecting GAS.

The association between history of PSGN and seropositivity to CRS, DRS and the $M$ peptides was examined. The proportion of individuals with past history of PSGN was the same $(56 \%)$ for those with and those without antibodies to CRS. However, there was a significant association between DRS seropositivity and history of PSGN (Fig. 2); 71 (65\%) of 109 DRS seropositive subjects had a recorded history of PSGN as opposed to $46 \%$ among the DRS seronegative subjects $(\mathrm{p}=0.009)$. There was no correlation between antibodies to any of the M peptides tested and history of PSGN. Given that PSGN is highly endemic in this population, and the disease has been reported to occur in a subclinical to clinical disease ratio of 4 to 1 [25]; many individuals may have had PSGN without having the diagnosis made. Therefore, the associations of 


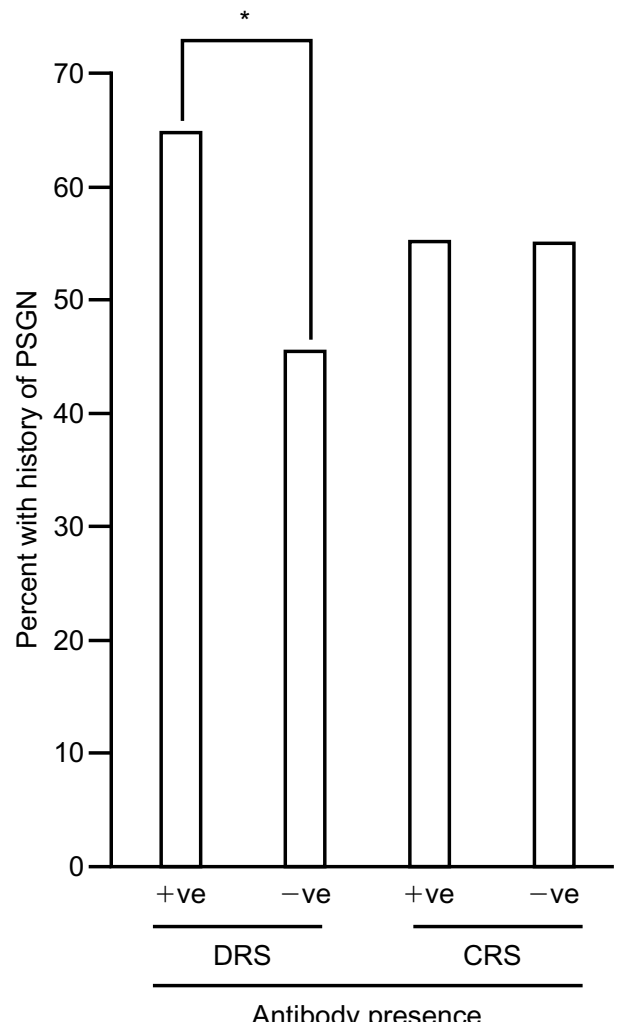

Fig. 2. Proportion of the population with a history of PSGN as determined by the presence or absence of antibodies to CRS and DRS. The data represent sera from 112 subjects with recorded PSGN history and 86 subjects who had no recorded history of PSGN; +ve and -ve represent sera with and without reactions to the antigens, CRS or DRS. *, Statistically significant $(\mathrm{p}=0.009)$.

serology with recorded history of PSGN may be underestimated in this study. The cut-off value used in this study is low, but analyses with higher cut-off points (OD 0.1 and 0.2 ) did not affect the relationships reported in this communication (data not shown). These results indicate either that seroreactivity to DRS is of pathological significance in PSGN or that it can act as a surrogate marker for diagnostic evaluation of history of PSGN.

The present study found that both CRS and DRS are immunogenic in natural infection and antibodies to these proteins are found among a large proportion of the population from a region highly endemic for PSGN. Profiles of antibody titres showed no correlation between seroreactions to CRS and M1/M57 peptides, or between seroreactions to DRS and M12/M55 peptides. However, there was a significant association between DRS seroreactivity and recorded history of PSGN. Further work in a PSGN non-endemic population is necessary to confirm this association, to test the predictive value for DRS antibodies in the diagnosis of PSGN and to investigate its role in PSGN pathogenesis.

This work was supported by the Australian National Health and Medical Research Council, the Australian Kidney Foundation and the
National Heart Foundation of Australia. We acknowledge the support and participation of the Tiwi Community, Tiwi project workers and the staff in the Health Clinic at Nguiu. We also thank Dr Wendy Hoy and Ms Bev Hayhurst, who initiated and co-ordinated the screening programme, and Drs Trenholme and Gardiner for reading the manuscript.

\section{References}

1. Dillon HC. Post-streptococcal glomerulonephritis following pyoderma. Rev Infect Dis 1979; 1: 935-945.

2. White AV, Hoy WE, McCredie DA. Childhood post-streptococcal glomerulonephritis as a risk factor for chronic renal disease in later life. Med J Aust 2001; 174: 492-496.

3. Lancefield RC. Persistance of type-specific antibodies in man following infection with group A streptococci. J Exp Med 1959; 110: 271-292.

4. Ferrieri P, Dajani AS, Chapman SS, Jensen JB, Wannamaker LW. Appearance of nephritis associated with type 57 streptococcal impetigo in North America. Longitudinal observations in a family. $N$ Engl J Med 1970; 283: 832-836.

5. Kaplan EL, Anthony BF, Chapman SS, Wannamaker LW. Epidemic acute glomerulonephritis associated with type 49 streptococcal pyoderma. I. Clinical and laboratory findings. Am J Med 1970; 48: 9-27.

6. Åkesson P, Sjoholm AG, Bjorck L. Protein SIC, a novel extracellular protein of Streptococcus pyogenes interfering with complement function. J Biol Chem 1996; 271: 1081-1088.

7. Hartas J, Sriprakash KS. Streptococcus pyogenes strains containing emm12 and emm55 possess a novel gene coding for distantly related SIC protein. Microb Pathog 1999; 26: $25-33$.

8. Scott JR, Cleary P, Caparon MG et al. New name for the positive regulator of the $\mathrm{M}$ protein of group A streptococcus. Mol Microbiol 1995; 17: 799.

9. Treser G, Semar M, McVicar M et al. Antigenic streptococcal components in acute glomerulonephritis. Science 1969; 163: 676-677.

10. Oliveira DB. Poststreptococcal glomerulonephritis: getting to know an old enemy. Clin Exp Immunol 1997; 107: 8-10.

11. Villarreal H, Fischetti VA, van de Rijn I, Zabriskie JB. The occurrence of a protein in the extracellular products of streptococci isolated from patients with acute glomerulonephritis. J Exp Med 1979; 149: 459-472.

12. Yoshizawa N, Oshima S, Takeuchi A et al. Experimental acute glomerulonephritis induced in the rabbit with a specific streptococcal antigen. Clin Exp Immunol 1997; 107: 61-67.

13. Yamakami K, Yoshizawa N, Wakabayashi K, Takeuchi A, Tadakuma T, Boyle MD. The potential role for nephritisassociated plasmin receptor in acute poststreptococcal glomerulonephritis. Methods 2000; 21: 185-197.

14. Parra G, Rodríguez-Iturbe B, Batsford S et al. Antibody to streptococcal zymogen in the serum of patients with acute glomerulonephritis. A multicentric study. Kidney Int 1998; 54: 509-517.

15. Cu GA, Mezzano S, Bannan JD, Zabriskie JB. Immunohistochemical and serological evidence for the role of streptococcal proteinase in acute post-streptococcal glomerulonephritis. Kidney Int 1998; 54: 819-826.

16. Rodríguez-Iturbe B. Epidemic poststreptococcal glomerulonephritis. Kidney Int 1984; 25: 129-136.

17. Hoe NP, Kordari P, Cole R et al. Human immune response to streptococcal inhibitor of complement, a serotype M1 group A Streptococcus extracellular protein involved in epidemics. $J$ Infect Dis 2000; 182: 1425-1436.

18. Martin DR, Sriprakash KS. Epidemiology of group A streptococcal disease in Australia and New Zealand. Australian Society for Microbiologists. Advances in Microbiology, vol 4. 1996.

19. Stata Corporation. 2000. Stata Statistical Software Programme, Release 6 edn.

20. Currie BJ, Carapetis JR. Skin infections and infestations in aboriginal communities in northern Australia. Australas $J$ Dermatol 2000; 41: 139-145.

21. Gardiner DL, Sriprakash KS. Molecular epidemiology of impetiginous group A streptococcal infections in aboriginal communities of northern Australia. J Clin Microbiol 1996; 34: $1448-1452$. 
22. Gardiner D, Hartas J, Hibble M, Goodfellow A, Currie B, Sriprakash KS. Molecular epidemiology of group A streptococcal infection in the Northern Territory of Australia. Adv Exp Med Biol 1997; 418: 317-321.

23. Stockbauer KE, Grigsby D, Pan X et al. Hypervariability generated by natural selection in an extracellular complementinhibiting protein of serotype M1 strains of group A
Streptococcus. Proc Natl Acad Sci USA 1998; 95: 3128-3133.

24. Daikos G, Weinstein L. Streptococcal bacteriostatic antibody in patients treated with penicillin. Proc Soc Exp Biol Med 1951; 78: $160-163$.

25. Rodríguez-Iturbe B, Rubio L, García R. Attack rate of poststreptococcal nephritis in families. A prospective study Lancet 1981; 1: 401-403. 